University of Windsor

Scholarship at UWindsor

2016

\title{
Is it appropriate to composite fish samples for mercury trend monitoring and consumption advisories?
}

\author{
Ken G. Drouillard \\ Nilima Gandhi \\ University of Windsor \\ Satyendra P. Bhavsar \\ Ontario Ministry of Environment \\ Sarah B. Gewurtz \\ University of Windsor \\ George B. Arhonditsis \\ University of Toronto - Erindale College
}

See next page for additional authors

Follow this and additional works at: https://scholar.uwindsor.ca/glierpub

Part of the Biochemistry, Biophysics, and Structural Biology Commons, and the Physical Sciences and Mathematics Commons

\section{Recommended Citation}

Drouillard, Ken G.; Gandhi, Nilima; Bhavsar, Satyendra P.; Gewurtz, Sarah B.; Arhonditsis, George B.; and Petro, Steve. (2016). Is it appropriate to composite fish samples for mercury trend monitoring and consumption advisories?. Environmental Science and Technology.

https://scholar.uwindsor.ca/glierpub/17

This Article is brought to you for free and open access by the Great Lakes Institute for Environmental Research at Scholarship at UWindsor. It has been accepted for inclusion in Great Lakes Institute for Environmental Research Publications by an authorized administrator of Scholarship at UWindsor. For more information, please contact scholarship@uwindsor.ca. 


\section{Authors}

Ken G. Drouillard, Nilima Gandhi, Satyendra P. Bhavsar, Sarah B. Gewurtz, George B. Arhonditsis, and Steve Petro 


\section{Is it appropriate to composite fish samples for mercury trend monitoring and consumption}

\section{advisories?}

Nilima Gandhi ${ }^{a}$, Satyendra P. Bhavsar ${ }^{a, b, c, *}$, Sarah B. Gewurtz ${ }^{c}$, Ken G. Drouillard ${ }^{c}$, George B. Arhonditsis ${ }^{a}$, Steve Petro ${ }^{b}$

${ }^{a}$ University of Toronto, Toronto, ON, Canada M1C 1A4

${ }^{\mathrm{b}}$ Ontario Ministry of the Environment and Climate Change, Toronto, ON, Canada, M9P 3V6

${ }^{\mathrm{c}}$ University of Windsor, 401 Sunset Avenue, Windsor, ON, Canada, N9B 3P4

* Corresponding author Tel: 1-416-327-5863; fax: 1-416-327-6519.

E-mail addresses: s.bhavsar@utoronto.ca or satyendra.bhavsar@ontario.ca (S.P. Bhavsar). 


\section{Abstract}

1 Monitoring mercury levels in fish can be costly because variation by space, time, and fish

2 type/size needs to be captured. Here, we explored if compositing fish samples to decrease

3 analytical costs would reduce the effectiveness of the monitoring objectives. Six compositing

4 methods were evaluated by applying them to an existing extensive dataset and examining their

5 performance in reproducing the fish consumption advisories and temporal trends. The methods

6 resulted in varying amount (average 34-72\%) of reductions in samples, but all (except one)

7 reproduced advisories very well (96-97\% of the advisories did not change or were one category

8 more restrictive compared to analysis of individual samples). Similarly, the methods performed

9 reasonably well in recreating temporal trends, especially when longer-term and frequent

10 measurements were considered. The results indicate that compositing samples within $5 \mathrm{~cm}$ fish

11 size bins or retaining the largest/smallest individuals and compositing in-between samples in

12 batches of 5 with decreasing fish size would be the best approaches. Based on the literature, the

13 findings from this study are applicable to fillet, muscle plug and whole fish mercury monitoring

14 studies. The compositing methods may also be suitable for monitoring Persistent Organic

15 Pollutants (POPs) in fish. Overall, compositing fish samples for mercury monitoring could result

16 in a substantial savings (approximately $60 \%$ of the analytical cost) and should be considered in

17 fish mercury monitoring, especially in long-term programs or when study cost is a concern.

19 Keywords: Mercury Hg; compositing/pooling; fish; monitoring; advisories; sensitive population 


\section{Graphical Abstract}

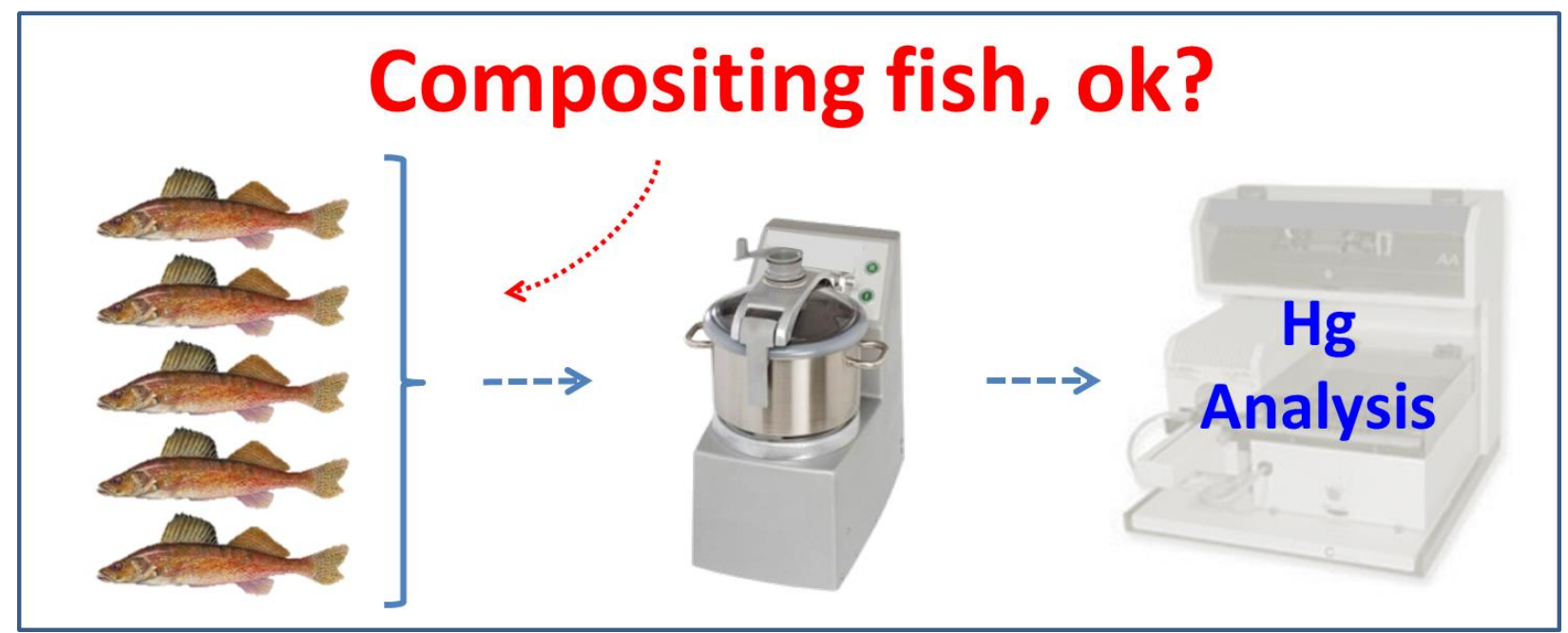

\section{Highlights}

- We test if compositing fish to decrease costs biases $\mathrm{Hg}$ monitoring

- Six compositing methods were assessed using an extensive dataset from Ontario

- Five methods reproduced advisories very well (96-97\% same or more stringent)

- Methods performed well in recreating temporal trends, especially for longer-terms

- Methods resulted in average 34-72\% reductions in samples and should be considered 


\section{Introduction}

Mercury is a contaminant of global concern (UNEP, 2013a). Virtually every fish in North

23 America, and possibly worldwide, contains mercury (Stahl et al., 2009; Depew et al., 2013;

24 Evers et al., 2013). Consumption of fish is generally a dominant route of human exposure to 25 mercury (UNEP/WHO, 2008). Mercury is responsible for the most number of restrictive fish 26 consumption advisories, at least in North America (e.g., USEPA, 2013a, b; OMOECC, 2015).

27 Due to spatial variation in fish mercury levels, location-specific advisories are typically provided 28 (e.g., USEPA, 2013a; OMOECC, 2015). Since mercury levels vary by fish species and size

29 (Gewurtz et al., 2011b), monitoring efforts to issue fish consumption advisories and track long-

30 term changes require collection and analysis of a variety of fish spanning their natural size range

31 (USEPA, 2013b). As a result, the total number of annual samples required to adequately monitor

32 fish mercury levels for numerous locations can range from hundreds to tens of thousands.

Due to analytical costs, most contaminant studies limit sample size by reducing the fish

34 species monitored, replication of samples, sampling frequency and/or study period; however,

35 these options are generally not suitable for agencies that rely on the data for long-term trend

36 monitoring and issuing of fish consumption advisories aimed at protecting human health

37 (Gewurtz et al., 2011a). Further, Article 19 of the recently formulated Minamata Convention on

38 Mercury requires parties to develop and improve geographically representative mercury

39 monitoring in environmental media, including fish (UNEP, 2013b). In less than a decade,

40 monitoring data will be called upon to assist in the implementation and evaluation of the

41 convention, which emphasizes the importance of improving monitoring efforts to optimize both

42 the quality of the programs as well as costs. 
To decrease program costs, combining multiple temporally or spatially discrete samples,

44 widely known as composites, has been suggested as an effective alternative to chemical analysis

45 on individual samples (USEPA, 2002; Gewurtz et al., 2011a). In addition to substantially

46 reducing analytical cost, the data collected through compositing samples can provide wider

47 temporal and spatial coverage without increasing the sample count. The analysis of data may

48 give more representative estimates of mean concentrations than can the same number of discrete

49 sampling, albeit at the cost of variability in the observations (USEPA, 2002).

There are several potential approaches to compositing fish contaminant monitoring

51 samples that incorporate different dimensions of the study, such as time (within/across years),

52 location, fish species, and fish size. The optimal compositing approach would be one that

53 reduces the total number of samples for analysis without compromising the objectives of the

54 monitoring program. In addition, the composite method chosen should follow assumptions that

55 correspond to the statistical analysis that is ultimately applied to the data. Several studies have

56 used compositing as a part of their designs for both organic and inorganic contaminants in all

57 media including biota (Rajagopal and Williams, 1989; Turle and Collins, 1992; Blomqvist, 2001;

58 Braune and Noble, 2009; Gewurtz et al., 2011a). However, to our knowledge, a comprehensive

59 study investigating the effectiveness of various compositing approaches for monitoring mercury

60 in fish is lacking in the literature, especially for programs designed to generate fish consumption

61 advice, where variability and the presence of outliers can affect overall risk (Gewurtz et al.,

62 2011a).

In this study, we evaluate six methods of compositing fish samples by examining their

64 performance if they would have been utilized instead of collecting $>220,000$ individual mercury

65 measurements for $>3000$ locations by the Province of Ontario, Canada over nearly 50 years. The 
66 effectiveness of the composite methods was evaluated by comparing the fish consumption

67 advisories and temporal trends from individual measurements (current sampling design) with

68 estimated composite values, calculated by averaging the individual measurements included in

69 each composite. The findings of the study determines whether a compositing method can

70 effectively minimize costs for regular, long term, large scale monitoring programs and set

71 advisories for fish consumption.

\section{2. Methods}

\subsection{Compositing Methods}

Fish mercury levels vary by species and size, and can change seasonally as well as over

76 time under the influence of a variety of internal and external factors, such as bioenergetics and

77 ambient water chemistry (Bhavsar et al., 2010; Azim et al., 2011; Gewurtz et al., 2011a; Stern et

78 al., 2012; Greenfield et al., 2013). As such, we opted to group species-specific samples collected

79 during the same sampling event within the composites.

There is a well-known relationship between mercury concentrations and fish size that is

81 typically described by the power-series regression (Gewurtz et al., 2011b). As such, similar

82 sized samples could be considered for creating a composite sample. However, the resultant fish

83 size range (i.e., maximum-minimum fish lengths) would likely be less than the regular,

84 individual measurements. This could result in trimming of a regression at the extreme ends, and

85 thereby loss of advisories for certain fish sizes. Alternatively, if one or two of the largest and/or

86 smallest individuals are retained with all other samples being composited, then the fish size 
87 range could be captured, and a power series regression between fish length and composited

88 mercury concentrations might be improved.

89 Compositing of 3, 5, 7, 10 or more samples have been used in many studies (Hites et al.,

90 2004; Carlson and Swackhamer, 2006; French et al., 2011; Pantazopoulos et al., 2013). Since a

91 collection of about 20 fish samples per species and sampling event over a possible maximum

92 size range is generally considered a preferred method for mercury monitoring (e.g., Gewurtz et

93 al., 2011a), compositing more than 5 samples (i.e., having less than four composites), may not be

94 sufficient for characterizing the fish size/mercury relationships. Alternatively, compositing

95 samples within a narrow size range (e.g., $35-40 \mathrm{~cm}, 40-45 \mathrm{~cm}$ and so on) regardless of the

96 number of samples within that size range may be appropriate as the impact on the fish

97 size/mercury relationship would likely be minimal.

98 Based on the above notes, we considered six compositing methods: (1) composite

99 samples in batches of five in the order of decreasing fish size (Figure 1a,b), (2) retain individual

100 samples for the largest and smallest fish and composite samples in between in batches of five in

101 order of decreasing fish size (Figure 1a,c), (3) retain the two largest and smallest individual

102 samples and composite the samples in between in batches of five in order of decreasing fish size

103 (Figure 1a,d), (4) retain the largest and smallest individual samples and composite the samples in

104 between in batches of three in order of decreasing fish size (Figure 1a,e), (5) retain the two

105 largest and smallest individual samples and composite the samples in between in batches of three

106 in the order of decreasing fish size (Figure 1a,f), and (6) composite samples within a $5 \mathrm{~cm}$ size

107 range (Figure 1a,g).

\subsection{Data Source}


110 from the individual fish measurements, assuming that the same mass of each fish is added to the

111 composite. For this purpose, we used an extensive and consistent fish mercury dataset

112 comprising 223,318 individual, widely varying measurements for skinless, boneless dorsal fillets

113 of $>10 \mathrm{~cm}$ fish of 66 fish species (Table S1) collected by the Ontario Ministry of the

114 Environment and Climate Change (OMOECC), Canada in partnership with the Ontario Ministry

115 of Natural Resources and Forestry and other agencies over nearly 50 years (1967-2014) from

$116>3000$ locations in the Province of Ontario, Canada, that spans $41^{\circ}$ to $56^{\circ} \mathrm{N}$ and $74^{\circ}$ to $95^{\circ} \mathrm{W}$

117 (Figure S1). The samples were analysed for total mercury using acid digestion and cold vapor

118 flameless atomic absorption spectroscopy as described in detail by Bhavsar et al. (2010). The

119 dataset contained 16,900 species/location/year combinations for 6,440 sampling events

120 (location/year) and varied widely (1 to 274) in the number of individual samples for a species in

121 a sampling event (species/location/year) (Figure S2).

\section{$122 \quad 2.3$ Statistical analysis}

The performance of each composite method in comparison to the regular, individual

124 measurements was evaluated based on its accuracy in reproducing the fish consumption

125 advisories as well as the direction and magnitude of the long-term temporal trends. As illustrated

126 in Figure S3, a power series regression was conducted for each of 16,900 species/location/year-

127 specific sampling events using the regular, individual measurements as well as the composite

128 values calculated using the six methods considered in this study. Using these total 118,300

129 power series regressions (i.e., 16,900 x 7), fish mercury levels were calculated at $5 \mathrm{~cm}$ intervals

130 for the available size range in each species-specific sampling event (Figure S3). These mercury

131 concentrations were used in calculating fish consumption advisories using the benchmarks for 
132 the general population and sensitive population (children and women of child-bearing age),

133 which is the standard method used by the Province of Ontario, Canada (Table S2, Figure S3).

134 Advisories for each $5 \mathrm{~cm}$ interval calculated using the six composite methods were compared

135 with those from the regular, individual measurements (Table S4), and classified into three

136 categories: 1) same, 2) more restrictive, and 3) less restrictive.

137 For a comparison of temporal trend analyses from the regular and composite methods,

138 rates of changes in fish mercury levels ( $\mu \mathrm{g} / \mathrm{g}$ decade) were calculated using the slope of the

139 linear relationship between year and mercury concentration standardised to a fish length. Since

140 the purpose is to compare rates from the regular and composite methods, appropriateness of a

141 linear regression is essentially a moot point (Azim et al., 2011). Since a temporal trend analysis

142 is typically conducted on a suitable indicator species with good monitoring data, four species,

143 namely Lake Trout (Salvelinus namaycush), Walleye (Sander vitreus), Northern Pike (Esox

144 Lucius) and Smallmouth Bass (Micropterus dolomieu), were considered. Mercury

145 concentrations standardized to $50 \mathrm{~cm}$ fish size were used. The standardization was conducted

146 using a power series regression $y=a x^{b}$, where $y$ is concentration in $\mu \mathrm{g} / \mathrm{g}, x$ is fish length in $\mathrm{cm}$,

147 and $a$ and $b$ are regression coefficients. The number of temporal trend rate estimates was

148 maximized by considering every combination of the start and end years as illustrated in Figure

149 S4. In total, 83,664 rates of fish mercury changes were calculated. All statistical analyses were 150 conducted in either Excel 2010 or R-3.2.0 for Windows ${ }^{\mathrm{TM}}$ (R Core Development Team, 2015).

\section{3. Results}

\section{$153 \quad 3.1$ Reductions in samples}


155 number of samples to be analysed for mercury (Figure 2). The composite methods 2 and 3

156 required retention of one and two extreme sized individual samples, respectively. As such, the

157 reductions in number of samples were less (method 2: 54/64\%; method 3: 40/50\%; Figure 2).

158 The methods 4 and 5 required compositing samples in the batches of 3 , compared to 5 for the

159 methods 2 and 3. As a result, reductions in the number of samples by implementing the methods

1604 and 5 were less (method 4: 45/53\%; method 5: 34/42\%; Figure 2). Although the composite

161 method 6 resulted in more variable (0-98\%) reductions in the samples because of its dependence

162 on number of samples in $5 \mathrm{~cm}$ fish size bins, overall reductions were similar to the method 2

163 (55/60\%; Figures 2, S5).

\subsection{Performance in reproducing advisories}

Seven sets of fish consumption advisories (regular plus six composite methods) were

166 calculated for each sampling event (species/location/year) as illustrated in Figure S3, and

167 compared as shown in Table S3. The resultant fish size ranges (minimum to maximum length)

168 for the composite method 1 were lower than from the regular, individual measurements for many

169 sampling events. In addition, method 1 produced one composite for each of 3,681 sampling

170 events with $\leq 5$ samples (Figure S2), resulting in no power series regression for an advisory

171 calculation. Therefore, about $35 \%$ of the advisories from method 1 were missing (Figure 3,

172 Table S3).

The advisories were calculated using power series regressions on fish size vs mercury

174 concentrations for each sampling event (location/year/species). The statistical significance of the 175 regressions was evaluated on the basis of their p-values. Since the composites were aimed at 
176 reducing the sample size, which is generally positively related to a p-value of a regression, it was

177 not surprising to observe lower statistical significance for regressions from a composite method

178 that produced a greater reduction in sample sizes (Figures 2, S6).

Overall, advisories for the general population from the methods 2 to 6 were largely (85-

$18091 \%$ ) similar to those from the regular, individual measurements (Figure 3, Table S4a). About

$1816-11 \%$ of the advisories were more restrictive, mostly by only one advisory category (Figure 3,

182 Table S4a). Only 3-4\% of the advisories were less restrictive, again mostly by only one advisory

183 category (Figure 3, Table S4a). The results for the sensitive population advisories were even

184 better (similar: 88-93\%; more restrictive 5-9\%; less restrictive 2-3\%; Figure 3, Table S4b).

The increasingly fewer reductions in the number of samples from the composite methods

1862 to 5 only marginally improved reproduction of the advisories (Figure 3 ). The performance of

187 the method 6 was similar to the method 4 and overall second best among the methods (Figure 3,

188 Table S4). Based on the reductions in the number of samples and performances in reproducing

189 the advisories, we focus further analysis and the following discussions on results for the general

190 population using the methods 2 and 6.

Next we examined if there was a pattern in the underestimation of mercury

192 concentrations and thereby less restrictive advisories from the composite methods that could be

193 linked to sample size, species, fish size class, and/or level of mercury. As shown in Tables S5-

194 S8, individually these four factors had minimal impact on the performance of the composite

195 methods 2 and 6. The only exception was that increasing fish size worsened the performance of

196 method 2, with relatively more cases of less restrictive advisories for large size categories within

197 individual species (Table S9). Nevertheless, there were only 3-4 combinations of species/size 
198 for which the total number of advisories were $>100$ and $>10 \%$ of the advisories were less

199 restrictive (Table S9). Similarly, there was no fish species-specific mercury concentration that

200 substantially affected the performance of the composite methods 2 and 6 (Table S10).

\subsection{Performance in reproducing temporal trends}

In this assessment, we examined if the nature of the mercury versus time slopes from the composite methods corresponded with the regular method. The composite methods resulted in

204 the same temporal trends as observed for the individual samples in most (90-94\%) cases (Figure

205 S7). The performances of the composite methods improved from 90-94\% to 94-96\% when cases

206 with a minimum time span of 15 years and 5 sampling years were considered, and to 95-97\%

207 when cases with a minimum time span of 15 years and 10 sampling years were considered

208 (Figure S7).

For a majority $(72-82 \%)$ of the cases, the rates of changes in fish mercury levels from the

210 composite methods were within a factor of two of the corresponding rates from the regular

211 method (Figure S8). Approximately 81-88\% of the rates were within a factor of three (Figure

212 S8). When cases with a minimum time span of 15 years and 5 sampling years were considered,

213 the percentages of cases improved to $81-88 \%$ for within a factor of two and $88-92 \%$ for within a

214 factor of three (Figure S8). The corresponding results for cases with a minimum time span of 15

215 years and 10 sampling years were better at 83-90\% and 89-93\%, respectively (Figure S8).

216 The performance of the composite methods in reproducing the rates of changes was also

217 evaluated for each of the four selected fish species. All composite methods provided the same

218 temporal trends for a majority (83-95\%) of the cases for all species (Figure S9). When cases

219 with a minimum time span of 15 years and 10 sampling years were considered, the percentages 
220 of cases improved to $97-100 \%$ for Lake Trout, Northern Pike and Walleye, and 86-90\% for

221 Smallmouth Bass (Figure S9). Likewise, performances of all methods in reproducing the rates

222 within a factor of two were comparatively similar for all species (Figure 4). When a more robust

223 dataset (cases with a minimum time span of 15 years and 10 sampling years) was considered, all

224 methods resulted in rates that were within a factor of three in $97-100 \%$ of the cases for Lake

225 Trout, Northern Pike and Walleye (Figure 4). The performance of the composite samples in 226 reproducing the rates of change for Smallmouth Bass was less (86-90\%) compared to the other

227 three species (Figure 4), indicating that Smallmouth Bass is the least preferred species for trend 228 monitoring when a composite method is utilised.

229 As expected, the composite methods that resulted in fewer reductions in the number of

230 fish mercury measurements provided better estimates of the rates of changes in the fish mercury

231 levels (Figures 2 and 4). Although reductions (55/60\%) in number of measurements from

232 method 6 were comparable to method 2 (54/64\%), method 6 provided better estimates of the

233 rates of change (Figure 2 and 4). Furthermore, the performance of method 6 was comparable to

234 the method 3, which consisted of relatively more mercury measurements (Figures 2 and 4). The

235 differences in the performance of the methods in reproducing the rates were minimal when cases

236 with a minimum time span of 15 years and 10 sampling years were considered (Figure 4).

238 4. Discussion

239 Composite sampling combines environmental samples or subsamples to form a new

240 sample on which chemical or biological analyses are performed. Compared to evaluating

241 individuals, composite sampling is beneficial as it decreases analytical cost by analyzing fewer 
242 samples and reduces/simplifies the sample handling process (USEPA, 2002). Composite

243 sampling is recommended when laboratory costs are substantially greater than field sampling

244 costs (USEPA, 2002). The collection of a few more fish samples at a particular location may not

245 substantially increase the field cost. However, the analytical savings associated with composite

246 sampling in long-term fish mercury monitoring and for issuance of fish consumption advisories

247 can be substantial, especially over time. For example, the approximately $60 \%$ reductions in

248 sample analyses in the OMOECC dataset used in this study would have resulted in

249 approximately 134,000 fewer fish mercury analyses over the 47 year period, which sums to

250 about $\$ 5,400,000$ (or $\$ 114,000$ per year) at an average rate of $\$ 40$ per sample. Similarly, about

$251 \$ 1,000,000$ could be saved for the dataset compiled by USGS from data collected by US states

252 (Hearn et al., 2006). Further, the composite sampling would have resulted in substantial saving in

253 other operational costs due to reduced number of samples to handle. Although the extent of cost

254 saving would depend on nature of the program (e.g., how many individual samples of which fish

255 species and sizes are presently analysed for mercury) and analytical cost, which has been

256 declining with advances in the analytical technology, the results presented in this study show that

257 savings can be achieved without any major impact on the quality of the advisories or temporal

258 trend assessments.

259 There are, however, some potential disadvantages of the composite sampling approach.

260 For example, composite sampling can result in a loss of information on extreme contamination

261 levels and variability. Although this is true in many cases, a composite method retaining one or

262 two largest and smallest individual samples as suggested in this study can potentially capture

263 extreme fish mercury levels due to the strong relationship of fish size and mercury concentration.

264 Although method 6 considered in this study may not preserve individual samples, a power series 
265 relationship between fish length and mercury indicates that compositing within a $5 \mathrm{~cm}$ fish size

266 bin would likely be able to provide values closer to the extreme levels. This could be a result of

267 the pattern in fish mercury levels, where even though there is a strong relationship between fish

268 length and mercury levels, it is not necessary that the biggest fish has the highest concentration

269 and the smallest fish has the lowest concentration likely due to differences in mercury levels in

270 spatially integrated fish samples. Compositing reduces sample size, and as such decreases

271 statistical power; however, statistical formulas can be used to derive composite size that results

272 in a sufficient power (Rohlf et al., 1996). The composite methods examined in this study also

273 resulted in some loss of statistical significance (Figure S6). Nevertheless, the methods

274 performed reasonably well in reproducing the advisories and temporal trends (Figures 3, 4, S7).

275 If contaminants other than mercury are also of interest, further evaluation of the

276 compositing methods may be necessary. For North America, other major contaminants of

277 concerns include persistent organic pollutants (POPs) for which compositing is often performed

278 (Hites et al., 2004; Gewurtz et al., 2011a) for studies focused on the health of fish themselves

279 and not on the generation of fish consumption advice. Gewurtz et al. (2011a) found compositing

280 fish samples appropriate for temporal trend monitoring of polychlorinated biphenyls (PCBs)

281 based on a limited evaluation of Lake Ontario lake trout measurements from different Canadian

282 and U.S. monitoring programs. However, their evaluation did not consider the impact of

283 compositing on the ability to detect outliers. It should be noted that the relationship between fish

284 length and POPs, such as PCBs, is much weaker than is typically observed for mercury (e.g.,

285 Gewurtz et al., 2011b). As such, compositing fish samples based on size categories (e.g., method

2866 in this study) may be less effective in capturing outliers for POPs. However, many agencies use

287 the "75\% rule" (i.e., the length of the smallest fish in a composite should be at least $75 \%$ of the 
length of the largest fish) for compositing fish samples for POP monitoring (e.g., Stahl et al.,

289 2009). The method 6 considered in this study will composite samples within a $5 \mathrm{~cm}$ size range

290 (Figure 1a,g) and follow the 75\% rule (except for fish smaller than $15 \mathrm{~cm}$, which are generally

291 not considered sport fish anyway). Similarly, the method 2 (and probably the other methods

292 considered) will also create composites (Figure 1a,c) that has a high potential to follow the 75\%

293 rule (Tables S11-S12), depending on the extent of sample collection by a program. As such, the

294 compositing methods and findings of this study may also be suitable for monitoring POPs in fish.

295 A reliable temporal trend analysis depends on within-year samples and duration of

296 monitoring (Sokal and Rohlf, 1995). Based on an exploratory analysis performed on data

297 collected by some Great Lakes biomonitoring programs and a comparison with the literature, it

298 was concluded that $>10$ years of monitoring with 10-15 samples per year is optimal to achieve

$29980 \%$ statistical power, which is typically considered adequate (Gewurtz et al., 2011a). This is

300 largely due to diminished sensitivity of a temporal trend analysis to start and end points when a

301 reasonable length of monitoring data is available (Gewurtz et al., 2011a). In this study, the

302 correspondence between the results from the regular and composite methods improved when a

303 longer time span and increased number of sampling years were considered (Figures 4, S7-S9).

304 As such, compositing samples may not be advisable for a short term assessment; however, the

305 accuracy of the regular method based on individual samples may also be poor.

In this study, we utilized skinless, boneless fillet mercury measurements to evaluate the

307 compositing methods. However, some monitoring programs use muscle plug or whole fish

308 measurements to track environmental conditions. Since fish fillet, muscle plug and whole fish

309 mercury measurements can be linked to one another (Baker et al., 2004; Peterson et al., 2005),

310 findings from this study should be applicable to muscle plug and whole fish mercury monitoring 
311 studies as well. The Ontario's fish contaminant monitoring is conducted exclusively in temperate

312 environments and thus the results from this study have broad applicability to other monitoring

313 programs in temperate latitudes. Although the in-depth analyses conducted on an extensive

314 dataset indicate that the findings should be applicable to tropical environment as well, further

315 work to verify these results in tropical environment may be warranted.

316 In summary, we explored the suitability of six composite methods for fish mercury

317 monitoring using an extensive dataset. The methods resulted in varying amount of reductions in

318 number of samples to be analyzed. In general, all compositing methods performed well for both

319 advisories on consumption of fish and temporal trend monitoring. The methods resulting in

320 lower reductions in sample count performed marginally better. Overall, compositing samples

321 would have resulted in a substantial cost savings for OMOECC (approximately $\$ 5.4 \mathrm{M}$ over 47

322 years assuming $60 \%$ sample reduction), and should be considered in fish mercury monitoring

323 especially in long-term extensive monitoring programs or when study cost is a concern.

325 Acknowledgements

326 We thank Ontario Ministry of the Environment and Climate Change, Canada, for the long-term

327 fish mercury dataset.

329 Supplementary Material

Additional 12 tables and 9 figures. This material is available free of charge via the

$331 \quad$ Internet at ??????

332

333 Notes The authors declare no competing financial interest. 


\section{References}

336 Azim, M., Kumarappah, A., Bhavsar, S., Backus, S., Arhonditsis, G., 2011. Detection of the spatiotemporal trends of mercury in Lake Erie fish communities: A Bayesian approach. Environ. Sci. Technol. 45, 2217-2226.

Baker, R.F., Blanchfield, P.J., Paterson, M.J., Flett, R.J., Wesson, L., 2004. Evaluation of nonlethal methods for the analysis of mercury in fish tissue. T. Am. Fish. Soc. 133, 568-

342 Bhavsar, S.P., Gewurtz, S.B., McGoldrick, D.J., Keir, M.J., Backus, S.M., 2010. Changes in mercury levels in Great Lakes fish between 1970s and 2007. Environ. Sci. Technol. 44, 3273-9.

Blomqvist, P., 2001. A proposed standard method for composite sampling of water chemistry and plankton in small lakes. Environ. Ecol. Stat. 8, 121-134.

Carlson, D.L., Swackhamer, D.L., 2006. Results from the US Great Lakes fish monitoring program and effects of lake processes on bioaccumulative contaminant concentrations. J.

Braune, B.M., Noble, D.G., 2009. Environmental contaminants in Canadian shorebirds. Environ. Monit. Assess. 148, 185-204. Great Lakes Res. 32, 370-385.

Depew, D.C., Burgess, N.M., Anderson, M.R., Baker, R., Bhavsar, S.P., Bodaly, R.A., Eckley, C.S., Evans, M.S., Gantner, N., Graydon, J.A., Jacobs, K., LeBlanc, J.E., St Louis, V.L., Campbell, L.M., 2013. An overview of mercury concentrations in freshwater fish species: a national fish mercury dataset for Canada. Can. J. Fish. Aquat. Sci. 70, 436-451. 
Evers, D.C., DiGangi, J., Petrlík, J., Buck, D.G., Šamánek, J., Beeler, B., Turnquist, M.A., Hatch, S.K., Regan, K., 2013. Global mercury hotspots: New evidence reveals mercury contamination regularly exceeds health advisory levels in humans and fish worldwide Biodiversity Research Institute, Gorham, Maine; IPEN, Göteborg, Sweden p. 20.

French, T.D., Petro, S., Reiner, E.J., Bhavsar, S.P., Jackson, D.A., 2011. Thirty-year time series of PCB concentrations in a small invertivorous fish (Notropis hudsonius): An examination of post-1990 trajectory shifts in the lower Great Lakes. Ecosystems 14, 415429.

Gewurtz, S.B., Backus, S.M., Bhavsar, S.P., McGoldrick, D.J., de Solla, S.R., Murphy, E.W., 2011a. Contaminant biomonitoring programs in the Great Lakes region: Review of approaches and critical factors. Environ. Rev. 19, 162-184.

Gewurtz, S.B., Bhavsar, S.P., Fletcher, R., 2011b. Influence of fish size and sex on mercury/PCB concentration: Importance for fish consumption advisories. Environ. Int. 32, 425-434.

Greenfield, B.K., Melwani, A.R., Allen, R.M., Slotton, D.G., Ayers, S.M., Harrold, K.H., Ridolfi, K., Jahn, A., Grenier, J.L., Sandheinrich, M.B., 2013. Seasonal and annual trends in forage fish mercury concentrations, San Francisco Bay. Sci. Total Environ. 444, 591601.

Hearn, P.P., Wente, S.P., Donato, D.I., Aguinaldo, J.J., 2006. EMMMA: A Web-based System for Environmental Mercury Mapping, Modeling, and Analysis. U.S. Geological Survey Open File Report 2006-1086, 13p.

Hites, R.A., Foran, J.A., Carpenter, D.O., Hamilton, M.C., Knuth, B.A., Schwager, S.J., 2004. Global assessment of organic contaminants in farmed salmon. Science 303, 226-9. 
OMOECC, 2015. 2015-2016 Guide to Eating Ontario Fish. Ontario Ministry of the Environment and Climate Change, Toronto, Ontario, Canada.

381 Pantazopoulos, P., Sawyer, J.M., Turyk, M.E., Diamond, M., Bhavsar, S.P., Mergler, D., Schantz, S., Ratnayake, N., Carpenter, D.O., 2013. Fatty acids in Great Lakes lake trout

Peterson, S.A., Van Sickle, J., Hughes, R.M., Schacher, J.A., Echols, S.F., 2005. A biopsy procedure for determining filet and predicting whole-fish mercury concentration. Arch. Environ. Con. Tox. 48, 99-107.

R Core Development Team, 2015. R: A language and environment for statistical computing. R Foundation for Statistical Computing, Vienna, Austria. .

Rajagopal, R., Williams, L.R., 1989. Economics of sample compositing as a screening tool in ground-water quality monitoring. Ground Water Monit. R. 9, 186-192.

Rohlf, F.J., Akcakaya, H.R., Ferraro, S.P., 1996. Optimizing composite sampling protocols. Environ. Sci. Technol. 30, 2899-2905.

Sokal, R.R., Rohlf, F.J., 1995. Biometry: the principles and practice of statistics in biological research, 3rd Edition ed. W. H. Freeman and Co., New York, NY, USA.

Stahl, L., Snyder, B., Olsen, A., Pitt, J., 2009. Contaminants in fish tissue from US lakes and reservoirs: a national probabilistic study. Environ. Monit. Assess. 150, 3-19.

Stern, G.A., Macdonald, R.W., Outridge, P.M., Wilson, S., Chetelat, J., Cole, A., Hintelmann, H., Loseto, L.L., Steffen, A., Wang, F., Zdanowicz, C., 2012. How does climate change influence arctic mercury? Sci. Total Environ. 414, 22-42.

Turle, R., Collins, B., 1992. Validation of the use of pooled samples for monitoring of contaminants in wildlife. Chemosphere 25, 463-9. 
402 UNEP, 2013a. Global Mercury Assessment 2013: Sources, Emissions, Releases and

403 Environmental Transport. UNEP Chemical Branch, UNEP Chemical Branch, Geneva, $404 \quad$ Switzerland.

405 UNEP, 2013b. Minamata Convention on Mercury. http://www.mercuryconvention.org.

406 UNEP/WHO, 2008. Guidance for identifying populations at risk from mercury exposure.

407 Geneva, Switzerland, p. 176.

408 USEPA, 2002. Guidance on choosing a sampling design for environmental data collection US

409 EPA, Washington, DC, p. 166.

410 USEPA, 2013a. Fish Consumption Advisories. USEPA. http://www2.epa.gov/fish-tech.

411 USEPA, 2013b. Guidance for Assessing Chemical Contaminant Data for Use In Fish Advisories.

412 USEPA. EPA 823-B-00-007.

413 http://water.epa.gov/scitech/swguidance/fishshellfish/techguidance.

414

415 
Figure 1: Illustration of six compositing methods considered in the study.

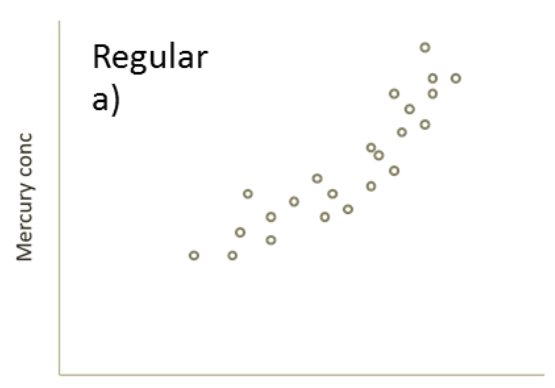

Individual measurements are shown in grey

Same coloured individuals belong to the same composite

\section{For this example sampling event}

\begin{tabular}{|l|c|c|c|c|c|c|c|}
\hline Method $\rightarrow$ & Reg & Comp 1 & Comp 2 & Comp 3 & Comp 4 & Comp 5 & Comp 6 \\
\hline Initial samples & 23 & 23 & 23 & 23 & 23 & 23 & 23 \\
\hline Final samples & 23 & 5 & 7 & 8 & 9 & 11 & 11 \\
\hline Reduction & $0 \%$ & $78 \%$ & $70 \%$ & $65 \%$ & $61 \%$ & $52 \%$ & $52 \%$ \\
\hline
\end{tabular}

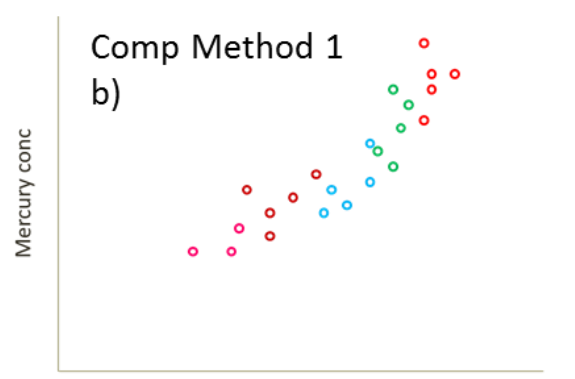

Comp Method 2

Comp Method 4

e)

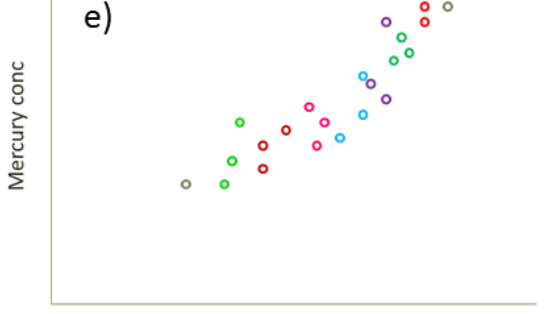

Fish length c)

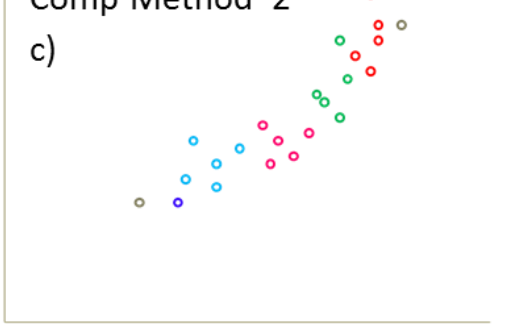

\section{Comp Method 5}

f)

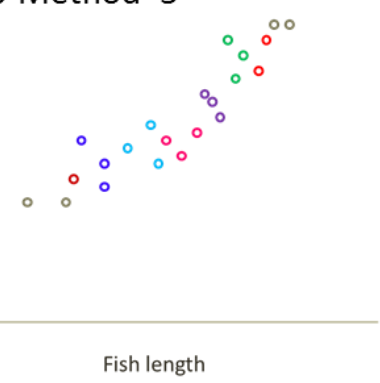

Comp Method 3

d)

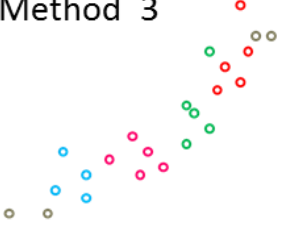

Comp Method 6

g)

$\because \because 00$

$\circ 0^{\circ}$

Fish length 
Figure 2: Overall reduction (\%) in number of samples per sampling event (location/year/species) analyzed in each of the six composite methods compared to the regular method of analyzing all individual fish samples for mercury.

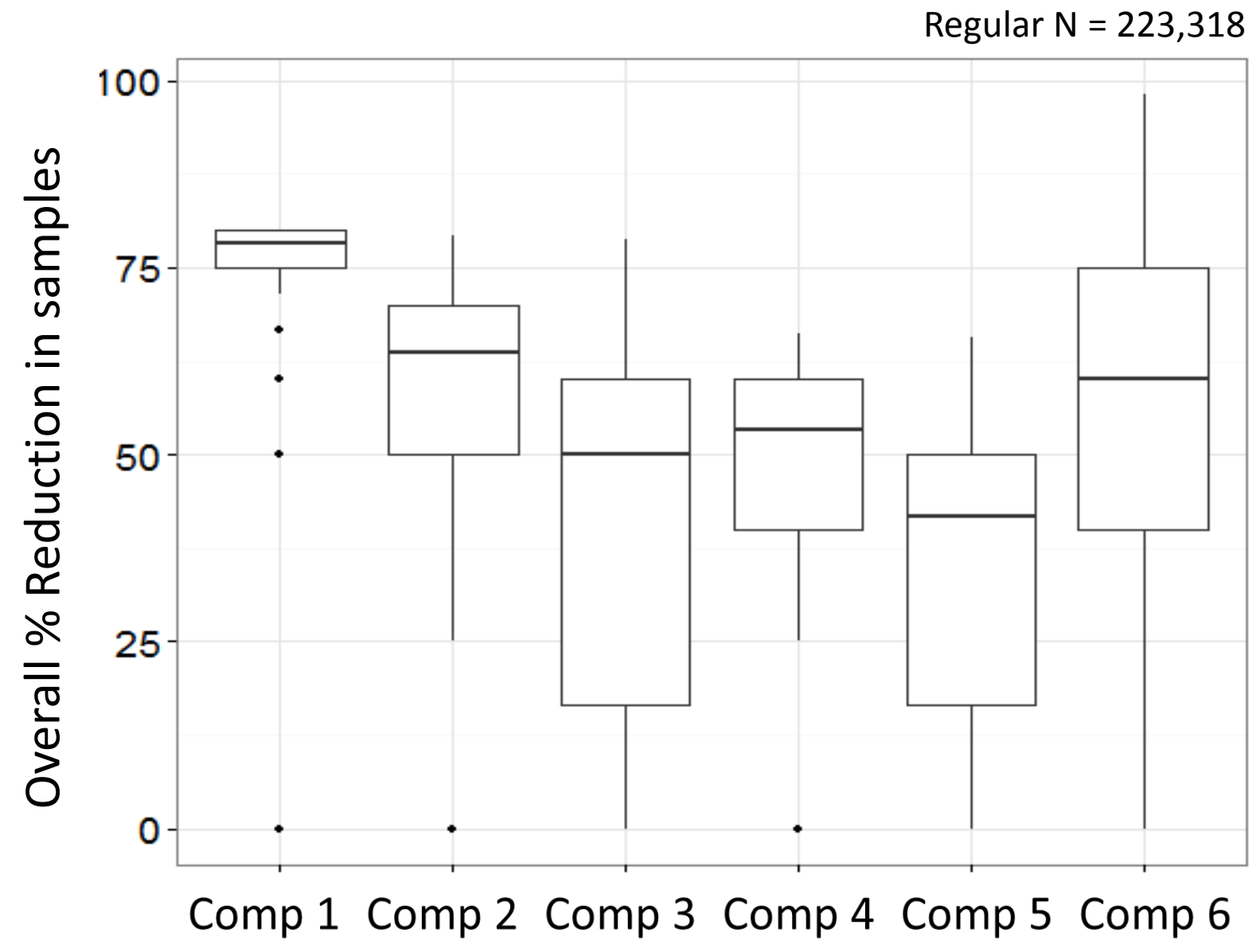


Figure 3: Comparison on fish consumption advisories for mercury for the general and sensitive populations using composite methods compared to the current OMOECC method of analyzing individual fish samples.

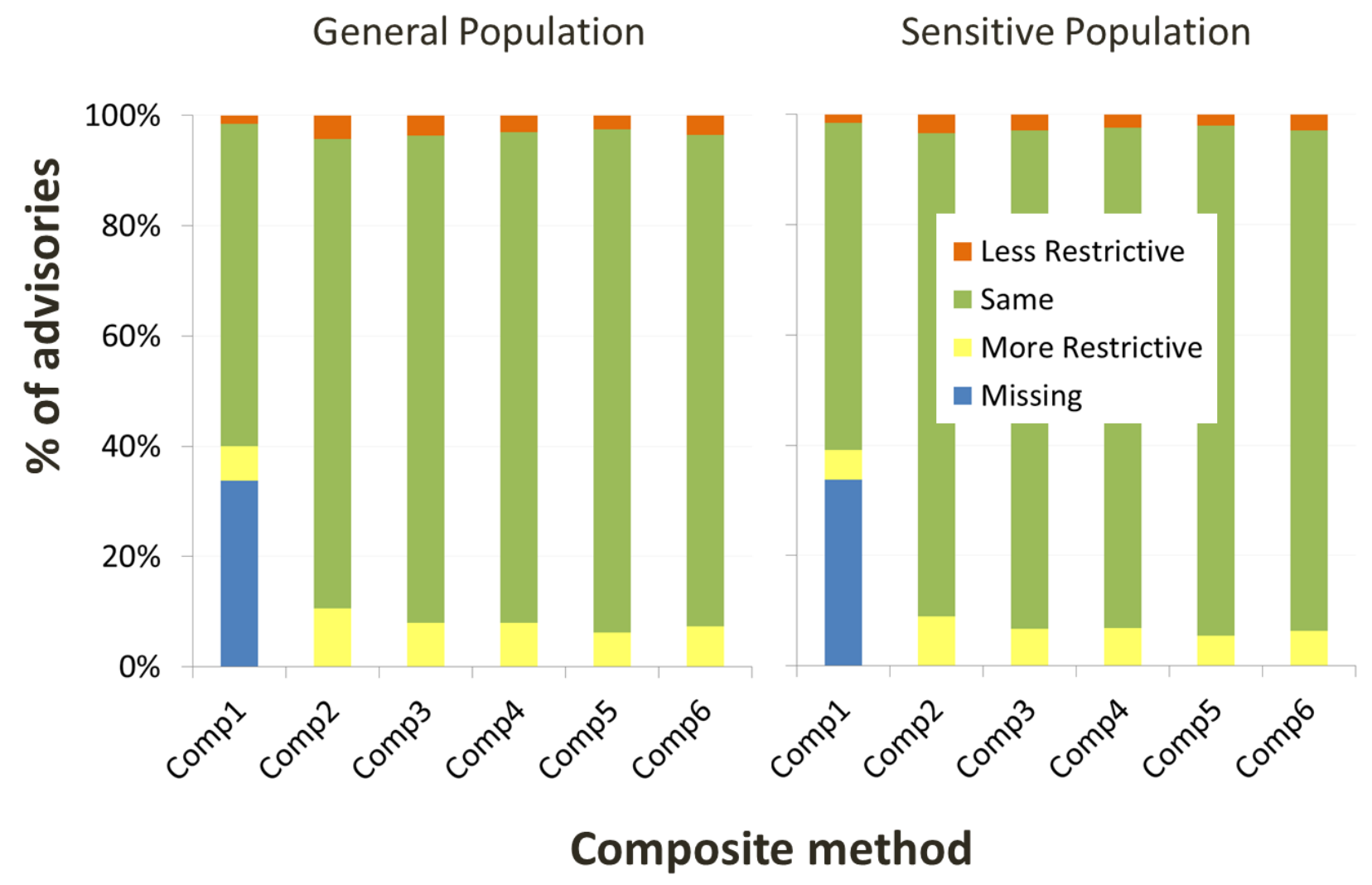


Figure 4: Comparison of rates of change in fish mercury levels of the six composite methods with those from the current OMOECC method of analyzing individual fish samples for mercury. The results have been presented as percentage of the total number of rate estimates within 2 and 3 times the corresponding rates from the current OMOECC method.

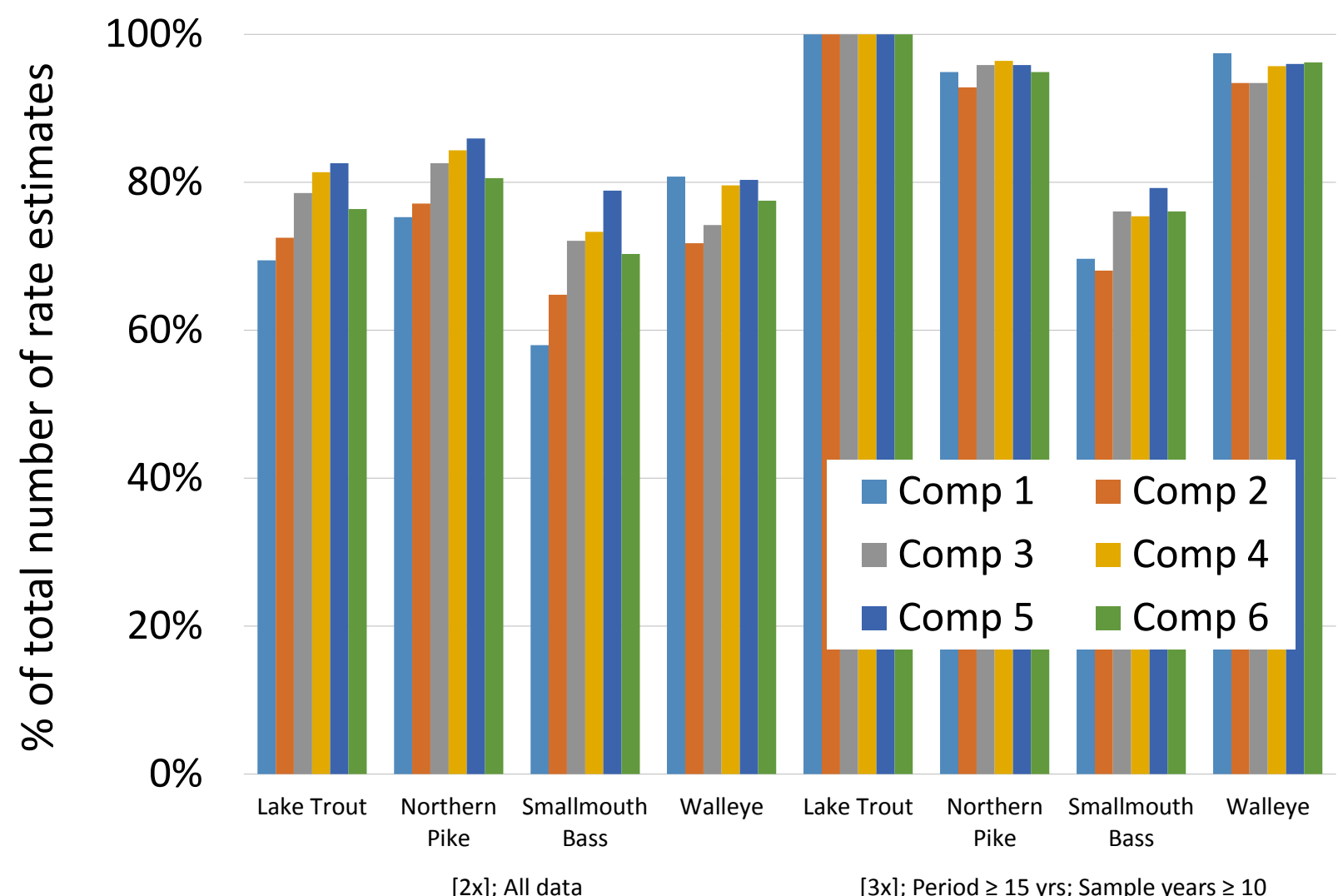

